OPEN ACCESS

Edited by:

Maciej Harat,

Franciszek Lukaszczyk Oncology

Centre, Poland

Reviewed by:

Eric Marvin,

Virginia Tech Carilion, United States

Brad E. Zacharia,

Penn State Milton S. Hershey Medical

Center, United States

*Correspondence:

Ting Zhang

zezht@zju.edu.cn

tThese authors have contributed equally to this work

Specialty section:

This article was submitted to Neuro-Oncology and Neurosurgical

Oncology,

a section of the journal

Frontiers in Neurology

Received: 29 November 2019

Accepted: 09 April 2020

Published: 13 May 2020

Citation:

Xin Z, Yao Y, Chen G, Wang L, Shu M,

Lv Q, Yu H and Zhang T (2020)

Fractionated Radiation Therapy for

Large and Giant Cavernous Sinus

Hemangioma: A Retrospective Study.

Front. Neurol. 11:355.

doi: 10.3389/fneur.2020.00355

\section{Fractionated Radiation Therapy for Large and Giant Cavernous Sinus Hemangioma: A Retrospective Study}

\author{
Zengfeng Xin ${ }^{1,2 t}$, Yihan Yao ${ }^{3+}$, Guodi Chen ${ }^{1}$, Liancong Wang ${ }^{1}$, Meibao Shu ${ }^{1}$, Qinghua $L v^{3}$, \\ Haifeng $\mathrm{Yu}^{4}$ and Ting Zhang ${ }^{1,3 *}$
}

${ }^{1}$ Department of Radiation Oncology, Zhejiang University School of Medicine, The Second Affiliated Hospital, Zhejiang University, Hangzhou, China, ${ }^{2}$ Department of Orthopedic Surgery, Zhejiang University School of Medicine, The Second Affiliated Hospital, Zhejiang University, Hangzhou, China, ${ }^{3}$ Key Laboratory of Tumor Microenvironment and Immune Therapy of Zhejiang Province, Hangzhou, China, ${ }^{4}$ Department of Chemotherapy Center, Zhejiang Cancer Hospital, Hangzhou, China

Purpose: Surgical resection has been traditionally used as a treatment for cavernous sinus hemangioma $(\mathrm{CSH})$. However, this is usually difficult due to tumor vascularity and results in complications especially in large and giant $\mathrm{CSH}$ (volume $>20 \mathrm{~cm}^{3}$ ). Previous studies have reported that radiotherapy (RT) provides an alternative treatment modality for hemangiomas. However, the optimized dose and fractions which control $\mathrm{CSH}$ and also protect the cognitive function remain unclear. This study reports our experience in the management of symptomatic large and giant $\mathrm{CSH}$.

Methods: Fifty-four patients with symptomatic large $\left(20 \mathrm{~cm}^{3}<\right.$ tumor volume $\leq 40$ $\mathrm{cm}^{3}, 3-4 \mathrm{~cm}$ in diameter) and giant (tumor volume $>40 \mathrm{~cm}^{3},>4 \mathrm{~cm}$ in diameter) $\mathrm{CSH}$ were enrolled in a retrospective study between January 2007 and December 2018. The prescription dose to the target margin was $50 \mathrm{~Gy}$ in 25 fractions.

Results: The mean pre-RT tumor volume was $60.9 \mathrm{~cm}^{3}$ which ranged from 20.2 to $230.5 \mathrm{~cm}^{3}$. The clinical data obtained was analyzed retrospectively following a mean follow-up period of 35.0 months which ranged from 1 to 140 months. All patients experienced tumor shrinkage within 3 months after radiotherapy. There was an average mean tumor reduction of $79.7 \%$ (range, 48.4-98.5\%) with no patients experiencing tumor progression and recurrence. All the 54 patients experienced symptomatic improvement within 1 month to 12 months after radiotherapy. Within the entire follow up period, no patients experienced any form of permanent complications or symptomatic radiation toxicity. Neurocognitive impairment studies were conducted before and after radiotherapy on 28 patients while the studies were conducted after the last follow up in 40 patients. The cognitive function of all the participants had normal MoCA-scores of 28.25 pre-radiotherapy. The post-treatment MoCA-scores were also clinically stable (28.04, $p=0.78$ ), and the average MoCA-score did not show any decline until the last follow-up (27.61, $p=0.13$ ).

Conclusion: The optimal dose and fractions of radiotherapy treatment for symptomatic large and giant cavernous sinus hemangioma remain unclear. This study, therefore, used a marginal dose of 50 Gy in 25 fractions in radiotherapy and this was proven to be effective and relatively safe in the treatment of symptomatic large and giant CSHs.

Keywords: hemangioma, cavernous sinus, central nervous system, radiotherapy, cognitive function 


\section{INTRODUCTION}

Hemangioma is a benign vascular tumor which causes clinical symptoms due to its progressive tumor growth and large size (1). Cavernous sinus hemangioma ( $\mathrm{CSH})$ is a rare benign tumor arising from the cavernous sinus and constitutes $<3 \%$ of sellar or para sellar tumors, and tend to occur mostly in middle-aged females (2). CSHs can develop with no elaborate symptoms in the cavernous sinus (CS). Nonetheless, large $\left(20 \mathrm{~cm}^{3}<\right.$ tumor volume $\leq 40 \mathrm{~cm}^{3}, 3-4 \mathrm{~cm}$ in diameter) and giant (tumor volume $>40$ $\mathrm{cm}^{3},>4 \mathrm{~cm}$ in diameter) CSHs may present with a variety of neurologic symptoms, such as blurred vision, diplopia, headache, seizures, and neurological deficit (3). The fatality rate in the case of a rupture or bleeding is extremely high.

For this type of benign tumor, treatment can be achieved through the complete removal of $\mathrm{CSH}$. Currently, the available treatments for $\mathrm{CSH}$ include microsurgical resection, embolization, stereotactic radiosurgery (SRS) such as Gamma Knife radiosurgery (GKRS), and fractionated radiotherapy $(4,5)$. The resection of large and giant CSHs is challenging due to the complex natural anatomic structures around the tumor (6). Surgical management of this lesion is also associated with extremely high risks due to the possibilities of significant blood loss and cranial neuropathies, hence, it is difficult to achieve total resection. Despite the recent advances in neurosurgical techniques, the total resection rate of CSHs by surgical excision is $64 \%$, and cranial neuropathies-related symptoms deteriorate after surgical treatment in $\sim 71 \%$ of the patients $(7,8)$.

Similar to the high risks of surgical treatment, radiotherapy (RT) for CSHs management has been extensively studied and reported. For example, in 1999, a postoperative patient with a residual lesion after resection was treated using GKRS. The postoperative results showed that the tumor markedly decreased and the preoperative clinical symptoms improved (9). Radiosurgery is currently an optimal choice for primary or postoperative treatment of $\mathrm{CSH}$ since it has demonstrated favorable clinical results. However, radiosurgery such as GKRS is suitable for small lesions $(10,11)$. Yamamoto et al. described their experiences of the successful treatment of about 30 cases of CSHs using radiosurgery. In their study, the average tumor volume was $11.5 \mathrm{~cm}^{3}$ (12). Due to the high morbidity of radiation complications, the size and location of the tumor limit the usage of GKRS for large CSHs, especially those adjacent to critical anatomic structures. Large lesions, therefore, require a larger target area and this may also lead to critical complications during irradiation for example on normal structures such as cerebral tissue and important nerves. Therefore, the use of fractionated stereotactic irradiation is increasing. In a previous study, hypofractionated stereotactic radiotherapy (H-SRT) was proven to effectively reduce the volume of $\mathrm{CSH}$ without causing severe radiation complications, even in large tumors with a median tumor volume of $19.3 \mathrm{~cm}^{3}$ (the range was from $15.8 \mathrm{~cm}^{3}$ to $69.3 \mathrm{~cm}^{3}$ ) (13). In another study, 14 large cavernous sinus hemangioma (volume $>20 \mathrm{~cm}^{3}$ ) patients were treated using $\mathrm{H}$ SRT, and the reported reduction rate of the tumor volume was $77 \%$, and with no radiotherapy-induced complications observed. These studies have confirmed the effectiveness and safety of
H-SRT (14). The reduction in the damage of normal structures while decreasing the volume of neoplasms is the main biological benefit of fractionated RT (15). For the current study, to achieve favorable reduction as well as to protect the optic pathway and other normal tissue from irradiation, this study's regimen of RT was used to deal with large and giant CSHs. In this retrospective study, the experience of 54 patients with large and giant CSHs in our institution was described. Therefore, the aim of this study was to evaluate the efficacy and safety of our regimen of RT as a treatment modality for large and giant CSHs.

\section{METHODS AND MATERIALS}

\section{Patients}

Patients diagnosed with large and giant CSHs (tumor volume $>20$ $\mathrm{cm}^{3}$ ) and treated with RT at the Second Affiliated Hospital of Zhejiang University from January 2007 to December 2018 were recruited in the study. There were 54 patients diagnosed during the stipulated period and their characteristics are presented in Table 1. This study was approved by the Ethics Committee of the Second Affiliated Hospital of Zhejiang University School of Medicine (No. 2019-220).

\section{Diagnosis}

A brain magnetic resonance imaging (MRI) and/or computed tomography (CT) study was used as the primary diagnostic tool in all the patients. CSHs were mainly diagnosed based on some specific imaging characteristics using MRI where the CSHs had well-defined boundaries in the MRI images (16). On T1-weighted images, the tumor was uniformly hypointense compared with the brain parenchyma, and also showed significantly brighter hyperintensity on T2-weighted images. The lesions became significantly enhanced after a contrast agent injection (17). The diagnosis was confirmed through pathological diagnosis or typical neuroimaging diagnosis. In this study, a total of 5 patients were pathologically diagnosed, while 49 patients were diagnosed through MRI with or without digital subtraction angiography (DSA).

\section{Treatment Strategy}

The Eclipse treatment planning system was used to fuse the CT and MRI scan with enhanced T1-weighted sequence to improve the target identification. The gross tumor volume (GTV) was defined as the enhanced tumor area according to the images. Expansion of the GTV margin by $5 \mathrm{~mm}$ in all directions was used to obtain the planning target volume (PTV). As shown in Table 2, evaluation of the target coverage, dose heterogeneity, and conformity were used to assess the quality of the treatment plans. 6-MV X-ray was used to deliver a total dose of $50 \mathrm{~Gy}$ in 25 fractions in 45 patients. However, 5 patients received $46 \mathrm{~Gy}$ in 23 fractions, whereas 2 patients received 40 Gy in 20 fractions and 1 patient received $45 \mathrm{~Gy}$ in 15 fractions. Plan normalized at $100 \%$ prescription cover $95 \%$ target volume. RT was performed using intensity modulated three-dimensional treatment plan across several different non-coplanar fixed fields in a linear accelerator (Varian). 
TABLE 1 | Baseline demographic and clinical factors of patients with large and giant CSHs.

\begin{tabular}{|c|c|}
\hline Variable & Results \\
\hline Age at study media (range) & $50.2(22-66)$ \\
\hline Age at diagnosis media (range) & $47.8(22-64)$ \\
\hline Sex (Male: Female) & $42: 12$ \\
\hline Length of follow up (month range) & $35.0(1-140)$ \\
\hline Symptom & $N(\%)$ \\
\hline Blurred vision & $33(61.1)$ \\
\hline Headache & 18 (33.3) \\
\hline Vomit & $6(11.1)$ \\
\hline Ptosis & $3(5.6)$ \\
\hline Proptosis & 5 (9.3) \\
\hline Dizziness & $21(38.9)$ \\
\hline Vertigo & $4(7.4)$ \\
\hline Diplopia & $3(5.6)$ \\
\hline Hemiplegia & $1(1.9)$ \\
\hline Weakness of upper extremity & $1(1.9)$ \\
\hline Diagnosis & $N(\%)$ \\
\hline Biopsy & $5(9.3)$ \\
\hline $\mathrm{MRI}+\mathrm{DSA}$ & $5(9.3)$ \\
\hline MRI & $44(81.4)$ \\
\hline \multicolumn{2}{|l|}{ Tumor volume } \\
\hline Pre-treatment $\left(\mathrm{cm}^{3}\right)$ (range) & $60.9(20.2-230.5)$ \\
\hline Post-treatment $\left(\mathrm{cm}^{3}\right)$ (range) & $10.3(1.3-39.1)$ \\
\hline Volume reduction (\%) (range) & $79.7(48.4-98.5)$ \\
\hline Symptom changes after RT & $N(\%)$ \\
\hline Recovery & $51(94.4)$ \\
\hline Improved & $3(5.6)$ \\
\hline Stable & $0(0)$ \\
\hline Dose/Fraction (Gy/F) & $N(\%)$ \\
\hline $50 / 25$ & 45 (83.3) \\
\hline $46 / 23$ & $5(9.3)$ \\
\hline $40 / 20$ & $2(3.7)$ \\
\hline $45 / 15$ & $2(3.7)$ \\
\hline ECOG & $N(\%)$ \\
\hline 0 & $22(40.7)$ \\
\hline 1 & $25(46.3)$ \\
\hline 2 & $4(7.4)$ \\
\hline 3 & $2(3.7)$ \\
\hline 4 & $1(1.9)$ \\
\hline Education level & $N(\%)$ \\
\hline Completed College/University & 14 (25.9) \\
\hline Completed high school & $20(37.0)$ \\
\hline Less than high school & $15(27.8)$ \\
\hline No education & $5(7.3)$ \\
\hline
\end{tabular}

Pt. No., patient number; M, Male; F, Female; RT, radiotherapy; MRI, Magnetic Resonance Imaging; DSA, Digital subtraction angiography.

\section{Neurocognitive Function}

The Montreal Cognitive Assessment (MoCA) was used to assess the neurocognitive performance of the patients (18). This assessment results were presented in the form of a total score and seven scores in seven different aspects: naming, memory, attention, language, executive and visuospatial functioning, abstraction and orientation (score 0-30). In this study, a score of $<26$ was used to define neurocognitive impairment (NCI) (19).

\section{Follow Up Period}

The first follow-up was conducted 3 months after the completion of RT, and subsequent follow-ups were conducted annually after the treatment. During each follow-up, a questionnaire was administered to obtain the patients clinical status and any other relevant physical examination, as well as brain MRI or CT scan, were performed. Based on MRI or CT results, the Eclipse planning system (version 13.6, Varian Medical Systems, Palo Alto, CA, USA) was used to estimate the tumor volumes. The National Cancer Institute Common Terminology Criteria for Adverse Events version 4.0. was used to score and document all treatment-induced toxicities.

\section{Statistical Analysis}

Basic descriptive statistics were used to demonstrate the baseline characteristics of patients, treatment information, and other variables measured during treatment and the follow-up period. For binary results, univariable analyses were conducted using a logistic regression model whereas linear regression was used for continuous variables. $t$-tests were used to analyze the difference between pre-treatment mean MoCA scores and post-treatment mean MoCA scores. All the statistical analyses were performed using SPSS software, version 20.0.

\section{RESULTS}

\section{Patients Characteristics and Treatment Modalities}

A total of 54 patients with $\mathrm{CSH}$ and treated between January 2007 and December 2018 were included in the study. As shown in Table 1, the majority of the patients were female $(77.8 \%, 42)$ with a median age of 47.8 years old (age range was from 22 to 64 years) at diagnosis. Prior to RT, five patients received operations, 4 of them underwent lesion biopsy while another one received partial resection. The most common initial symptoms among the patients were blurred vision $(61.1 \%, 33)$, dizziness $(38.9 \%, 21)$, headache $(33.3 \%, 18)$, vomit $(11.1 \%, 6)$, proptosis $(9.3 \%, 5)$, ptosis $(5.6 \%, 3)$, vertigo $(7.4 \%, 4)$, and weakness in upper extremities $(1.9 \%, 1)$. The mean hemangioma volume was $61.3 \mathrm{~cm}^{3}$ (the range was between 20.2 to $230.5 \mathrm{~cm}^{3}$ ) before treatment.

Treatment characteristics of each patient are presented in Table 2. The RT was performed at the median 95\% isodose line. The mean tumor coverage receiving the entire prescribed dose was $94.7 \%$ (ranging from 91.7 to $97.8 \%$ ), and the mean conformity index was 0.82 (ranging from 0.76 to 0.88 ). In addition, the average tumor maximum dose was $53.36 \mathrm{~Gy}$ (ranging from 46.97 to $58.09 \mathrm{~Gy}$ ), while the mean minimal dose of the tumor was $45.74 \mathrm{~Gy}$ (ranging from 37.60 to $49.15 \mathrm{~Gy}$ ). Furthermore, the average maximum dose irradiating to the ipsilateral optic nerve, optic chiasm, and brainstem were 43.58Gy (range was between 21.03 and 53.60Gy), 46.67 Gy (range was between 21.18 and $53.62 \mathrm{~Gy}$ ), and $44.19 \mathrm{~Gy}$ (range was between 
TABLE 2 | Treatment characteristics and dose volume analyses of 54 patients.

\begin{tabular}{|c|c|c|c|c|c|c|c|c|c|c|}
\hline Pt. No. & $\begin{array}{l}\text { Maximum tumor } \\
\text { dose (Gy) }\end{array}$ & $\begin{array}{l}\text { Minimum tumor } \\
\text { dose (Gy) }\end{array}$ & $\begin{array}{l}\text { Average tumor } \\
\text { dose (Gy) }\end{array}$ & $\begin{array}{l}\text { Prescription tumor } \\
\text { dose }(G y / F)\end{array}$ & $\mathrm{Cl}$ & $\mathrm{HI}$ & Coverage & $\begin{array}{l}\text { Maximum optic } \\
\text { nerve dose (Gy) }\end{array}$ & $\begin{array}{l}\text { Maximum optic } \\
\text { chiasm dose (Gy) }\end{array}$ & $\begin{array}{c}\text { Maximum brainstem } \\
\text { dose (Gy) }\end{array}$ \\
\hline 1 & 57.25 & 47.96 & 52.67 & $50 / 25$ & 0.82 & 1.13 & 94.7 & 43.41 & 51.49 & 39.12 \\
\hline 2 & 52.88 & 48.11 & 51.57 & $50 / 25$ & 0.77 & 1.06 & 92.1 & 21.03 & 21.18 & 25.11 \\
\hline 3 & 56.09 & 48.83 & 53.72 & $52 / 26$ & 0.77 & 1.07 & 97.1 & 21.63 & 47.39 & 49.90 \\
\hline 4 & 57.69 & 48.12 & 53.91 & $52 / 26$ & 0.83 & 1.09 & 93.9 & 44.12 & 52.13 & 50.12 \\
\hline 5 & 55.43 & 47.98 & 52.16 & $50 / 25$ & 0.81 & 1.10 & 94.3 & 43.17 & 51.78 & 49.93 \\
\hline 6 & 54.04 & 43.25 & 51.43 & $50 / 25$ & 0.79 & 1.08 & 93.8 & 52.53 & 52.08 & 52.01 \\
\hline 7 & 50.39 & 43.76 & 47.85 & $46 / 23$ & 0.78 & 1.09 & 93.7 & 48.67 & 48.24 & 47.79 \\
\hline 8 & 46.89 & 38.21 & 43.17 & $40 / 20$ & 0.76 & 1.12 & 92.6 & 42.78 & 43.76 & 43.42 \\
\hline 9 & 53.58 & 47.72 & 51.46 & $50 / 25$ & 0.83 & 1.07 & 94.0 & 48.15 & 43.32 & 47.59 \\
\hline 10 & 49.76 & 42.55 & 46.75 & $45 / 15$ & 0.76 & 1.11 & 91.7 & 47.23 & 44.84 & 46.19 \\
\hline 11 & 55.39 & 41.68 & 51.86 & $50 / 25$ & 0.79 & 1.09 & 95.2 & 46.37 & 42.22 & 40.69 \\
\hline 12 & 50.23 & 40.21 & 46.21 & $44 / 22$ & 0.81 & 1.08 & 94.2 & 40.12 & 37.54 & 38.76 \\
\hline 13 & 55.25 & 47.66 & 53.62 & $50 / 25$ & 0.84 & 1.12 & 95.2 & 44.41 & 51.12 & 41.14 \\
\hline 14 & 46.97 & 41.91 & 46.01 & $45 / 25$ & 0.81 & 1.04 & 95.5 & 46.65 & 46.97 & 46.21 \\
\hline 15 & 49.65 & 37.60 & 46.42 & $45 / 25$ & 0.80 & 1.10 & 95.0 & 46.30 & 46.69 & 47.18 \\
\hline 16 & 55.04 & 43.97 & 51.99 & $50 / 25$ & 0.86 & 1.09 & 94.6 & 42.19 & 50.66 & 29.41 \\
\hline 17 & 54.11 & 46.80 & 51.37 & $50 / 25$ & 0.88 & 1.07 & 95.3 & 52.60 & 51.93 & 50.88 \\
\hline 18 & 54.95 & 45.20 & 52.03 & $50 / 25$ & 0.78 & 1.10 & 95.6 & 53.00 & 53.62 & 53.40 \\
\hline 19 & 55.25 & 47.93 & 51.59 & $50 / 25$ & 0.87 & 1.08 & 94.5 & 42.12 & 49.23 & 35.97 \\
\hline 20 & 53.88 & 48.91 & 50.87 & $50 / 25$ & 0.87 & 1.09 & 92.4 & 25.76 & 50.53 & 28.43 \\
\hline 21 & 55.09 & 48.97 & 53.53 & $50 / 25$ & 0.79 & 1.02 & 97.8 & 31.33 & 46.69 & 44.90 \\
\hline 22 & 56.73 & 49.15 & 51.17 & $50 / 25$ & 0.84 & 1.09 & 95.2 & 44.12 & 40.83 & 45.65 \\
\hline 23 & 52.78 & 48.08 & 51.96 & $50 / 25$ & 0.83 & 1.11 & 94.4 & 42.87 & 50.68 & 49.44 \\
\hline 24 & 53.14 & 45.45 & 51.68 & $50 / 25$ & 0.75 & 1.04 & 94.8 & 52.12 & 51.48 & 51.33 \\
\hline 25 & 51.49 & 44.32 & 48.21 & $50 / 25$ & 0.79 & 1.12 & 94.7 & 45.67 & 47.32 & 48.23 \\
\hline 26 & 53.67 & 46.21 & 49.17 & $50 / 25$ & 0.78 & 1.08 & 94.6 & 42.45 & 44.74 & 42.68 \\
\hline 27 & 54.15 & 47.72 & 51.96 & $50 / 25$ & 0.84 & 1.11 & 94.4 & 48.21 & 45.39 & 45.21 \\
\hline 28 & 55.99 & 47.55 & 49.98 & $50 / 25$ & 0.86 & 1.09 & 94.7 & 46.11 & 43.23 & 43.13 \\
\hline 29 & 54.97 & 41.68 & 51.86 & $50 / 25$ & 0.82 & 1.09 & 95.1 & 43.37 & 42.52 & 46.69 \\
\hline 30 & 51.04 & 46.21 & 49.92 & $50 / 25$ & 0.84 & 1.05 & 94.6 & 41.14 & 34.54 & 35.56 \\
\hline 31 & 54.89 & 47.69 & 53.52 & $50 / 25$ & 0.85 & 1.11 & 95.3 & 43.41 & 49.42 & 40.74 \\
\hline 32 & 47.65 & 41.91 & 47.01 & $45 / 25$ & 0.83 & 1.07 & 95.4 & 44.65 & 45.97 & 46.11 \\
\hline 33 & 48.89 & 42.60 & 47.42 & $45 / 25$ & 0.81 & 1.11 & 95.1 & 44.30 & 46.59 & 46.18 \\
\hline 34 & 55.32 & 45.97 & 51.43 & $50 / 25$ & 0.87 & 1.08 & 94.7 & 42.21 & 45.66 & 34.41 \\
\hline 35 & 53.96 & 47.80 & 51.57 & $50 / 25$ & 0.88 & 1.09 & 95.4 & 52.70 & 52.93 & 52.85 \\
\hline 36 & 55.02 & 46.20 & 52.07 & $50 / 25$ & 0.79 & 1.11 & 95.5 & 52.00 & 53.62 & 52.58 \\
\hline 37 & 56.91 & 47.56 & 52.37 & $50 / 25$ & 0.84 & 1.12 & 94.6 & 43.41 & 46.49 & 41.12 \\
\hline 38 & 53.12 & 47.54 & 51.47 & $50 / 25$ & 0.79 & 1.07 & 94.1 & 26.73 & 50.08 & 28.11 \\
\hline
\end{tabular}


৪ 둥

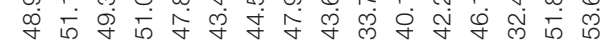

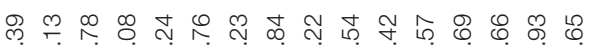
辛

ช 든

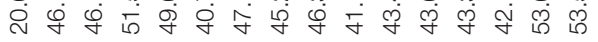

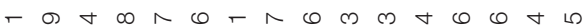

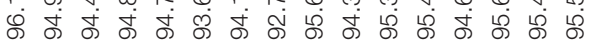

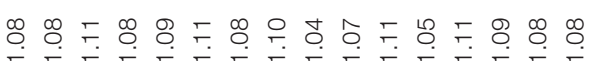

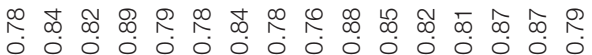
$\overline{0}$

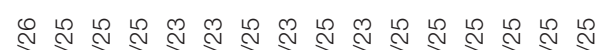
तิ ठे ठे ठ্

ㄷำ ชิ่

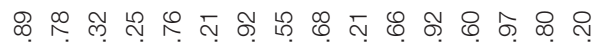

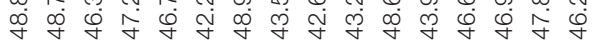

ㅇำ ம
25.11 and $53.67 \mathrm{~Gy}$ ), respectively. Figure 1 shows one of the treatment plans.

\section{Imaging Response}

The average follow-up period for all the patients was 35.0 months (ranging from 1 to 140 months). The MRI or CT images demonstrated significant tumor shrinkage in all the patients and the tumors control rate was $100 \%$. Shrinkage of the CSHs was seen immediately after the completion of RT. The tumor volume was about $65.7 \%$ of their initial volume at the end of the RT and kept decreasing during the follow-up period. Three months post RT, the masses had reduced in size to $42.9 \%$ of the original volume (Figures 3A,B). At 1-year post RT, they reduced further to $29.7 \%$ and $23.6 \%$ at 2 years post-RT. In some patients, the tumor sizes remained stable without any change in size while on others, they kept decreasing even after 5 years follow-up (Figures 2A,B). The MRI results indicated a $98.7 \%$ tumor volume reduction 2 years after RT (Figure 1). However, no transient tumor enlargement was observed in participants after RT.

\section{Clinical Response}

All the patients had favorable neurological outcomes after the treatment. The neurologic symptoms changes after treatment are shown in Table 1. All pretreatment symptoms had recovered or improved within 1 month to 1 year after RT. Fifty-one patients (94.4\%) experienced symptoms recovery after the treatment, while three patients (5.6\%) had their symptoms improved which lasted for many years. During the final follow-up, all the participating patients showed favorable RT neurological outcomes. Only one patient expressed feelings of dizziness.

\section{Toxicity}

There was no patient who suffered severe acute or delayed complications associated with RT. All the observed side effects were mild and transient. Grade 1 nausea and anorexia developed in only two patients (3.7\%). While Grade 2 nausea occurred in three patients $(5.6 \%)$ during the course of RT. However, these symptoms of RT-related side effects were managed with corticosteroids and they completely disappeared. MRI showed that some patients had encephaledema about 2 years after RT, but they had no clinical symptoms. There were no other acute or early delayed side effects from RT observed both during the treatment and follow-up period.

\section{Neurocognitive Assessment}

Among 54 participating patients, 40 patients had the neurocognitive test done and they completed the MoCA at least once. Among them, 28 patients had the neurocognitive impairment study before and after RT while 40 patients did the study during the last follow-up. The cognitive functions of the participants were in the normal range (total MoCA-scores of 28.25) pre-RT. In comparison, post-treatment MoCA-scores were clinically stable $(28.04, p=0.78)$ and the average MoCAscore was not decreased at the last follow-up (27.61, $p=0.13$ ) (Figure 3C). 
A

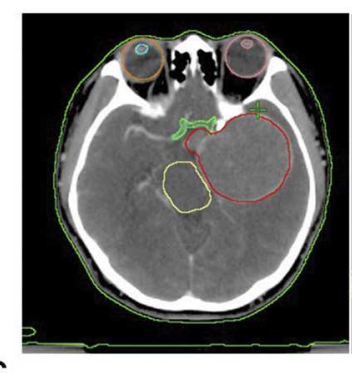

C
20150421

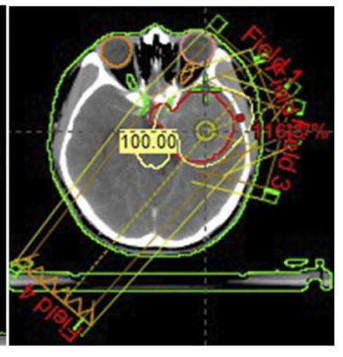

B

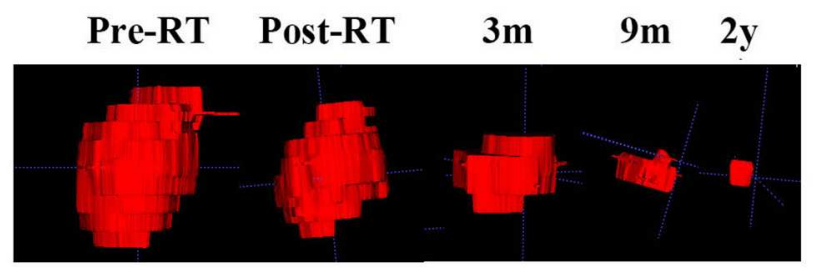

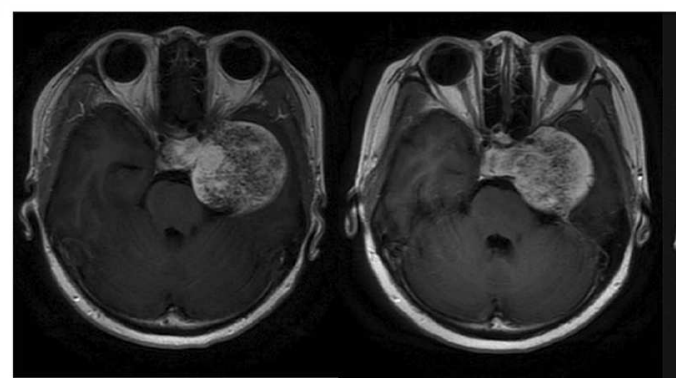

Pre-RT

Post-RT

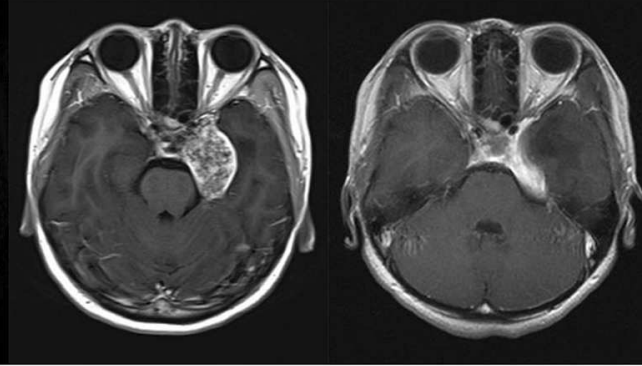

$3 m$

9m

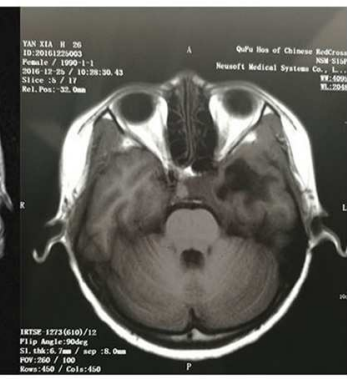

$2 y$

FIGURE 1 | A mass occupying the left cavernous sinus of a 24-year-old woman (patient 1) was shrunk gradually after RT. (A) Treatment plan based on CT scan. (B) The 3D imaging of the mass before RT and at a different time of follow up after RT. (C) MRI scan before RT, the day after RT completion and 3 months, 9 months, and 2 years after $\mathrm{RT}$.

\section{DISCUSSION}

In this retrospective study, the efficacy of radiotherapy as a treatment modality for patients with large and giant CSHs was evaluated. RT of a marginal dose of $50 \mathrm{~Gy}$ in 25 fractions at the median $95 \%$ prescription isodose line was used. The results of this study demonstrated an average $79.7 \%$ tumor reduction. Moreover, the pretreatment symptoms such as visual disturbance and headache could be cured or improve after RT. In all the patients there was no severe radiotherapy-induced complications observed during the treatment and neither during the subsequent follow-up period. The cognitive function of the patients did not also change during the entire treatment and follow-up period. These results revealed the efficacy and safety of our regimen of RT for large and giant CSHs.

Surgery is a traditional treatment for CSHs, however, in spite of there being several advanced surgical techniques, it is difficult to completely remove large and giant CSHs through surgery. This is due to the complex and critical anatomic structures in the cavernous sinus and the possible risk of excessive bleeding during the operation. Previous studies have demonstrated a low total surgical removal rate and high morbidity of complications after surgery in CSH $(1,17)$. Majority of the cases reported arose from the neurosurgery department.

Radiotherapy has become an emerging approach for CHS treatment for patients with residual tumors after surgery as well as those with great intraoperative risk. This is due to its efficacy and safety compared with surgical treatment. As early as the 1980s, radiosurgery was used for the treatment of high surgical risk CSH and has been successfully used as adjuvant therapy in $\mathrm{CSH}$ patients having residual lesions after surgical removal $(9,20)$. Subsequently, more studies have been conducted on the function of radiotherapy for CSHs due to its remarkable tumor shrinkage effect. Gamma Knife surgery is one of the radiotherapy techniques which has been proven to be safe and is being used for primary as well as postoperative management of CSH patients. A retrospective study analyzing the effect of Gamma Knife surgery in patients with CSH reported an average tumor volume reduction of $82 \%$, and most of the pretreatment symptoms had been improved (21). In another meta-analysis, by Wang et al. 59 patients with a mean followup period of 49.2 months (range between 6 and 156 months) was studied. The results supported stereotactic radiosurgery as an alternative treatment for CSHs because of its reduction in traditional surgery-associated complications (22). However, the treatment outcomes of GKRS were associated with the tumor volume, underdosed tumor volume and total treated volume (23). Single-shot radiosurgery was reported to be effective for patients with small- or middle-sized CSHs. The high risk of radiation-induced optic neuropathy (RION) limits the usage of single-shot stereotactic radiosurgery for the patients with large CSHs (23). However, for large and giant cavernous sinus hemangiomas, Gamma Knife radiosurgery may not the optimal treatment. Previous studies, indicate that the risk of radiationinduced complications such as cranial nerve palsies and cognitive damage, could be higher if fractionation is not performed (24). 
A

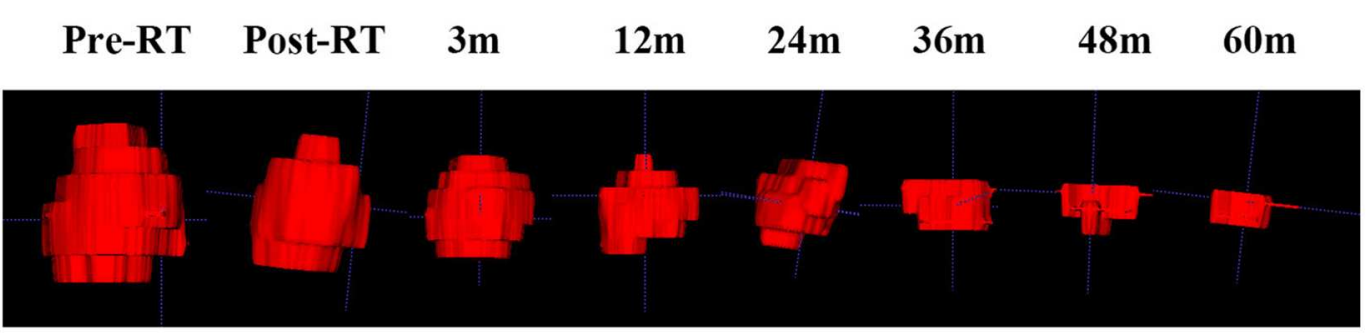

B

Pre-RT

Post-RT

$3 m$

$12 \mathrm{~m}$

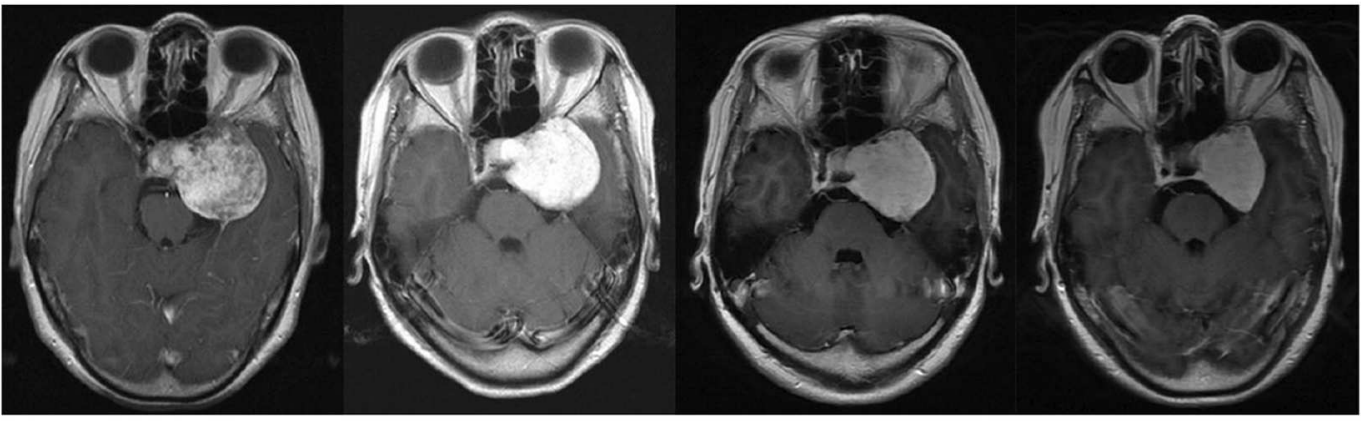

$24 m$

$36 \mathrm{~m}$

$48 m$

$60 \mathrm{~m}$

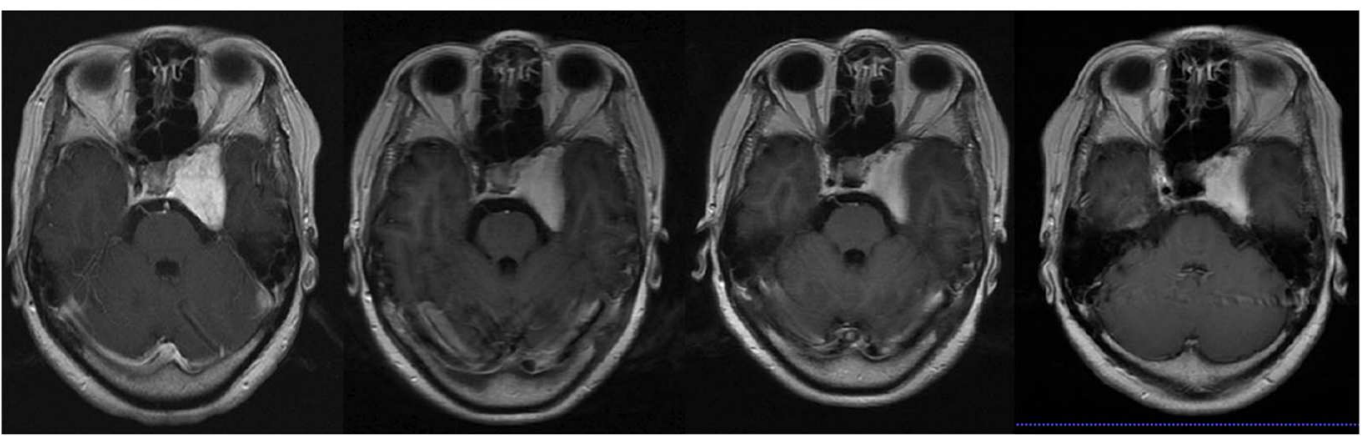

FIGURE 2 | CSH of patient 7 kept decreasing even 5 years later. (A) The 3D imaging of the mass before RT and at different times of follow up after RT. (B) MRI scan before RT, the day after RT completion and at a different time of follow up after RT.

Wang X. reported hypofractionated stereotactic radiosurgery using CyberKnife with $21 \mathrm{~Gy}$ in 3 fractions or $22 \mathrm{~Gy}$ in 4 fractions for the treatment of giant CSHs (volume $>40 \mathrm{~cm}^{3}$, $>4 \mathrm{~cm}$ in diameter) in 31 patients. Compared with pretreatment tumor volume, the results demonstrated a median tumor volume reduction of $88.1 \%(62.3-99.4 \%)$ during the last follow-up (3), with a mean follow-up period of 30 months. However, there was a concern about cognitive impairment as a long-term side effect.

It has been reported that cognitive impairment is one of the radiation encephalopathies which may significantly affect a patient's quality of life $(25,26)$. Radiation-induced cognitive impairment can be divided as acute, early delayed and late effects, with late effects being considered to be irreversible (27). The mechanism of the effect of RT on cognitive function has been reported in previous studies (25). Radiotherapy induces vascular damage and changes in white matter, including demyelination and coagulative necrosis (28). One of the essential regions of cognition is the hippocampus which has been found to be damaged after radiation. In some preclinical studies, neurogenesis inhibition following brain radiotherapy was demonstrated which caused cognitive impairment $(29,30)$. In this study, we followed up on the long-term effect of RT, however, no cognitive impairment was observed.

Fractionated stereotactic radiotherapy is another alternative treatment modality for large and giant CSHs especially those showing great risk through traditional surgery and radiosurgery. However, studies about conventional radiotherapy performed for $\mathrm{CSH}$ are rare with most of those available being case reports. In these relevant studies, the radiation dose varied from $30 \mathrm{~Gy}$ to $50 \mathrm{~Gy}$ (31). A phase II study focusing on hypofractionated stereotactic radiotherapy for large (volume> $20 \mathrm{~cm}^{3}$ ) CSH reported a mean of $77 \%$ tumor volume reduction 
A

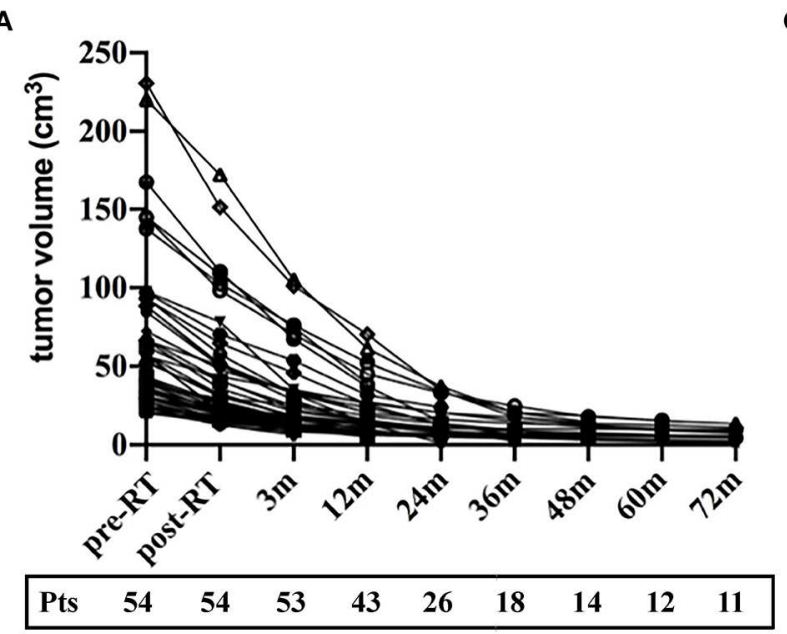

B

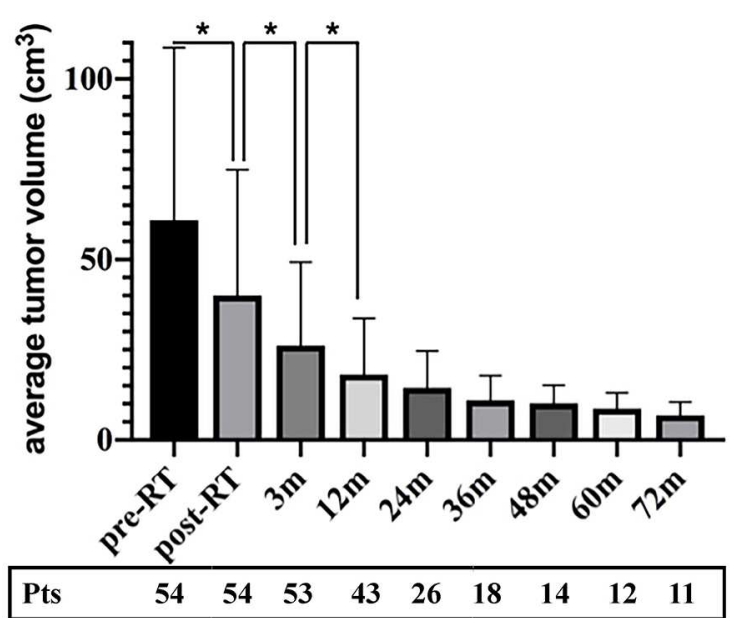

C

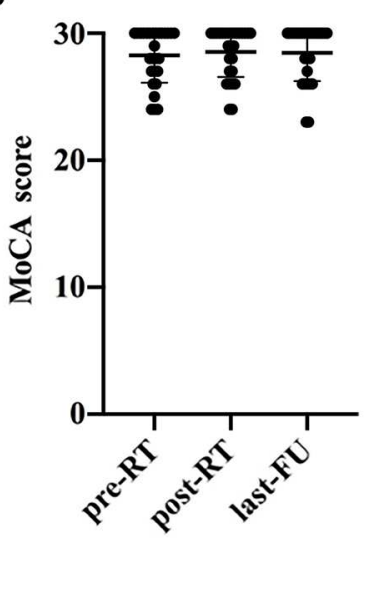

FIGURE 3 | The tumor volume reduced over the entire follow-up period without transient enlargement in all the patients. (A) The volume of the CSHs decreased in all patients. (B) The percentage of tumor volume during the follow-up intervals to their initial volume. (C) The MoCA score of participants at three-time points (pre-treatment, post-treatment and the last follow-up).

(range, 44-99\%), 14 patients included, and the radiotherapy dose was $21 \mathrm{~Gy}$ delivered in 3 fractions, however the mean follow-up period was 15 months (range, 6-36 months) (14). The current study focused on large and giant CSHs and has a longer follow-up period. As a benign tumor, long-term survival is expected, and it is necessary to minimize the toxicity of RT such as $\operatorname{RION}(32,33)$.

This study, however, had a number of limitations. First, it was a retrospective and single-centered study with a small number of patients to make a definitive conclusion. Therefore, this needs further research and the inclusion of larger sample size in a multicenter study. Besides, for observing long-term cognitive deficits, the follow-up time period was relatively short, longer and more extensive follow-up is needed. The assessment of neurocognitive function involves many factors which have an effect on the MoCA scores, therefore, it was difficult to balance the factors due to the limited sample size. In addition, MoCA score is not the optimal tool for neurocognitive assessment in brain tumor patients. Furthermore, this study did not include a control group, and both the patients and investigators were aware of the treatment, which may exert influence on symptom reporting. Overall, further research on radiotherapy on $\mathrm{CSH}$ is required.

\section{CONCLUSIONS}

MRI helps clinicians to make a diagnosis in large and giant cavernous sinus hemangioma, but the optimal treatment for symptomatic CSHs remain unclear. Although longer follow-up is needed, RT using a marginal dose of $50 \mathrm{~Gy}$ in 25 fractions was found to be effective and relatively safe in the treatment of symptomatic large and giant CSHs.

\section{DATA AVAILABILITY STATEMENT}

The datasets used and analyzed during the current study are available from the corresponding author on reasonable request. 


\section{ETHICS STATEMENT}

This study was approved by the Ethics Committee of the Second Affiliated Hospital of Zhejiang University School of Medicine (No. 2019-220).

\section{AUTHOR CONTRIBUTIONS}

TZ and YY designed this study and wrote the manuscript. GC, LW, and MS analyzed the clinical data and participated in the manuscript writing. TZ, YY, and GC interpreted the clinical data. HY and QL read and interpreted the radiology images. YY and LW collected the clinical data of surgery and radiotherapy. ZX and YY conceived the study and wrote the manuscript. All authors had read and approved the final manuscript.

\section{REFERENCES}

1. Linskey ME, Sekhar LN. Cavernous sinus hemangiomas: a series, a review, and an hypothesis. Neurosurgery. (1992) 30:101-8. doi: 10.1097/00006123-199201000-00018

2. Bakhsheshian J, Zada G, Pham MH. Cavernous sinus hemangioma: rare vascular tumor of cavernous sinus. World Neurosurg. (2018) 110:4323. doi: 10.1016/j.wneu.2017.11.177

3. Wang X, Zhu H, Knisely J, Mei G, Liu X, Dai J, et al. Hypofractionated stereotactic radiosurgery: a new treatment strategy for giant cavernous sinus hemangiomas. J Neurosurg. (2018) 128:60-7. doi: 10.3171/2016.10.JNS16693

4. Khan AA, Niranjan A, Kano H, Kondziolka D, Flickinger JC, Lunsford LD. Stereotactic radiosurgery for cavernous sinus or orbital hemangiomas. Neurosurgery. (2009) 65:914-8 discussion 8. doi: 10.1227/01.NEU.0000356987.98197.71

5. Lee CC, Sheehan JP, Kano H, Akpinar B, Martinez-Alvarez R, MartinezMoreno N, et al. Gamma knife radiosurgery for hemangioma of the cavernous sinus. J Neurosurg. (2017) 126:1498-505. doi: 10.3171/2016.4.JNS152097

6. Yin YH, Yu XG, Xu BN, Zhou DB, Bu B, Chen XL. Surgical management of large and giant cavernous sinus hemangiomas. J Clin Neurosci. (2013) 20:128-33. doi: 10.1016/j.jocn.2012.01.050

7. Escudeiro GP, Leal RTM, Leal da Silveira R, Landeiro JA. Cavernous sinus hemangioma: surgical aspects. World Neurosurg. (2019) 123:156. doi: 10.1016/j.wneu.2018.11.162

8. Khattar NK, Adams SW, Schaber AS, White AC, Al Ghamdi M, Hruska RT, et al. Endoscopic endonasal surgery for the resection of a cavernous hemangioma with a sellar extension. Cureus. (2018) 10:e3663. doi: 10.7759/cureus.3663

9. Iwai Y, Yamanaka K, Nakajima H, Yasui T. Stereotactic radiosurgery for cavernous sinus cavernous hemangioma-case report. Neurol Med chir (Tokya). (1999) 39:288-90. doi: 10.2176/nmc.39.288

10. Anqi X, Zhang S, Jiahe X, Chao Y. Cavernous sinus cavernous hemangioma: imaging features and therapeutic effect of Gamma knife radiosurgery. Clin Neurol Neurosurg. (2014) 127:59-64. doi: 10.1016/j.clineuro.2014.09.025

11. Tripathi M, Batish A, Kumar N, Ahuja CK, Oinam AS, Kaur R, et al. Safety and efficacy of single-fraction gamma knife radiosurgery for benign confined cavernous sinus tumors: our experience and literature review. Neurosurg Rev. (2018) 43:27-40. doi: 10.1007/s10143-018-0975-8

12. Yamamoto M, Kida Y, Fukuoka S, Iwai Y, Jokura H, Akabane A, et al. Gamma knife radiosurgery for hemangiomas of the cavernous sinus: a seven-institute study in Japan. J Neurosurg. (2010) 112:772-9. doi: 10.3171/2009.6.JNS08271

13. Haghighi N, Seely A, Paul E, Dally M. Hypofractionated stereotactic radiotherapy for benign intracranial tumours of the cavernous sinus. J Clin Neurosci. (2015) 22:1450-5. doi: 10.1016/j.jocn.2015.03.026

14. Wang X, Liu X, Mei G, Dai J, Pan L, Wang E. Phase II study to assess the efficacy of hypofractionated stereotactic radiotherapy in patients with

\section{FUNDING}

This work was supported by grants from The Natural Science Foundation of Zhejiang Province (LY12H10006, LY17C080002, LY19C080002, LY19C080001), The Department of Health of Zhejiang Province (2017KY365, 2018KY424) and The National Natural Science Foundation of China (31200652).

\section{ACKNOWLEDGMENTS}

The authors would like to thank Dr. Guangwei Yu, Dr. Chao Li, Dr. Kui Wu from Department of Radiation Oncology and Dr. Bing Pan from Department of psychiatry department, The Second Affiliated Hospital, Zhejiang University School of Medicine.

large cavernous sinus hemangiomas. Int J Radiat Oncol Biol Phys. (2012) 83:e223-30. doi: 10.1016/j.ijrobp.2011.12.051

15. Park S, Yoon SM, Lee S, Park JH, Song SY, Lee SW, et al. Role of fractionated radiotherapy in patients with hemangioma of the cavernous sinus. Radiat Oncol J. (2017) 35:268-73. doi: 10.3857/roj.2017.00199

16. Meincke J, Lutzen N, Doostkam S, Urbach H. Teaching neuroimages: "Filling out" in cavernous hemangioma of the cavernous sinus. Clin Neuroradiol. (2018) 28:137-8. doi: 10.1007/s00062-017-0607-7

17. Zhou LF, Mao Y, Chen L. Diagnosis and surgical treatment of cavernous sinus hemangiomas: an experience of 20 cases. Surg Neurol. (2003) 60:31-6 discussion 6-7. doi: 10.1016/S0090-3019(03)00190-3

18. McDowell LJ, Ringash J, Xu W, Chan B, Lu L, Waldron J, et al. A cross sectional study in cognitive and neurobehavioral impairment in long-term nasopharyngeal cancer survivors treated with intensity-modulated radiotherapy. Radiother Oncol. (2019) 131:179-85. doi: 10.1016/j.radonc.2018.09.012

19. Hsiao KY, Yeh SA, Chang CC, Tsai PC, Wu JM, Gau JS. Cognitive function before and after intensity-modulated radiation therapy in patients with nasopharyngeal carcinoma: a prospective study. Int J Radiat Oncol Biol Phys. (2010) 77:722-6. doi: 10.1016/j.ijrobp.2009.06.080

20. Shibata S, Mori K. Effect of radiation therapy on extracerebral cavernous hemangioma in the middle fossa. Report of three cases. J Neurosurg. (1987) 67:919-22. doi: 10.3171/jns.1987.67.6.0919

21. Chou CW, Wu HM, Huang CI, Chung WY, Guo WY, Shih $\mathrm{YH}$, et al. Gamma knife surgery for cavernous hemangiomas in the cavernous sinus. Neurosurgery. (2010) 67:611-6 discussion 6. doi: 10.1227/01.NEU.0000378026.23116.E6

22. Wang X, Mei G, Liu X, Pan L, Wang E. The role of stereotactic radiosurgery in cavernous sinus hemangiomas: a systematic review and meta-analysis. J Neurooncol. (2012) 107:239-45. doi: 10.1007/s11060-0110753-8

23. Kuo JS, Chen JC, Yu C, Zelman V, Giannotta SL, Petrovich Z, et al. Gamma knife radiosurgery for benign cavernous sinus tumors: quantitative analysis of treatment outcomes. Neurosurgery. (2004) 54:1385-93 discussion 93-4. doi: 10.1227/01.NEU.0000124750.13721.94

24. Levegrun S, Hof H, Essig M, Schlegel W, Debus J. Radiation-induced changes of brain tissue after radiosurgery in patients with arteriovenous malformations: dose/volume-response relations. Strahlenther Onkol. (2004) 180:758-67. doi: 10.1007/s00066-004-1266-6

25. Jacob J, Durand T, Feuvret L, Mazeron JJ, Delattre JY, Hoang-Xuan K, et al. Cognitive impairment and morphological changes after radiation therapy in brain tumors: a review. Radiother Oncol. (2018) 128:2218. doi: 10.1016/j.radonc.2018.05.027

26. Meyers CA, Brown PD. Role and relevance of neurocognitive assessment in clinical trials of patients with CNS tumors. J Clin Oncol. (2006) 24:13059. doi: 10.1200/JCO.2005.04.6086 
27. Ali FS, Hussain MR, Gutierrez C, Demireva P, Ballester LY, Zhu JJ, et al. Cognitive disability in adult patients with brain tumors. Cancer Treat Rev. (2018) 65:33-40. doi: 10.1016/j.ctrv.2018. 02.007

28. Valk PE, Dillon WP. Radiation injury of the brain. AJNR Am J Neuroradiol. (1991) 12:45-62.

29. Rola R, Raber J, Rizk A, Otsuka S, VandenBerg SR, Morhardt DR, et al. Radiation-induced impairment of hippocampal neurogenesis is associated with cognitive deficits in young mice. Exp Neurol. (2004) 188:31630. doi: 10.1016/j.expneurol.2004.05.005

30. Mizumatsu S, Monje ML, Morhardt DR, Rola R, Palmer TD, Fike JR. Extreme sensitivity of adult neurogenesis to low doses of X-irradiation. Cancer Res. (2003) 63:4021-7.

31. Grosu AL, Nieder C. Stereotactic fractionated radiotherapy for recurrent capillary hemangioma of the cavernous sinus. Strahlenther Onkol. (2006) 182:179-82. doi: 10.1007/s00066-006-1473-4

32. Mayo C, Martel MK, Marks LB, Flickinger J, Nam J, Kirkpatrick J. Radiation dose-volume effects of optic nerves and chiasm. Int J Radiat
Oncol Biol Phys. (2010) 76(3 Suppl):S28-35. doi: 10.1016/j.ijrobp.2009. 07.1753

33. Leavitt JA, Stafford SL, Link MJ, Pollock BE. Long-term evaluation of radiation-induced optic neuropathy after single-fraction stereotactic radiosurgery. Int J Radiat Oncol Biol Phys. (2013) 87:524-7. doi: 10.1016/j.ijrobp.2013.06.2047

Conflict of Interest: The authors declare that the research was conducted in the absence of any commercial or financial relationships that could be construed as a potential conflict of interest.

Copyright (c) 2020 Xin, Yao, Chen, Wang, Shu, Lv, Yu and Zhang. This is an openaccess article distributed under the terms of the Creative Commons Attribution License (CC BY). The use, distribution or reproduction in other forums is permitted, provided the original author(s) and the copyright owner(s) are credited and that the original publication in this journal is cited, in accordance with accepted academic practice. No use, distribution or reproduction is permitted which does not comply with these terms. 